\title{
The Characterization of Dominating Region of Fracture (process region) around a Crack tip Based on the Concept of Mechanical Similarity and Atomic Mechanics
}

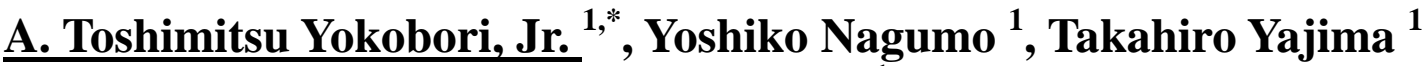 \\ Toshihito Ohmi ${ }^{1}$
}

${ }^{1}$ Department of Nanomechanics, Tohoku University, Sendai, 980-8579, Japan

* Corresponding author: yokobori@md.mech.tohoku.ac.jp

\begin{abstract}
Brittle fracture at a crack tip is considered to be caused within a specified local region, that is, process region. However, the determination of this scale has not yet been theoretically clarified. The difficulty of this determination will be due to the wide range scale analysis from the range of nano scale (atomic scale) to macro scale (crack size scale). In this paper, on the basis of the atomic mechanics using super atom and hybrid method of the fractal concept which concerns self-similarity with the proposed analysis of scale projection from macro to nano scales, the disturbed region of atom arrangements around a crack tip were clarified under the local stress field by crack and dislocation. This disturbed region was related to fracture dominating region (process region) which is in good agreement with the scale obtained from experimental consideration by R. Ritchie.
\end{abstract}

Keywords Process region, Mechanical similarity, Atomic mechanics, Brittle fracture

\section{Introduction}

The fracture criterion of materials is considered to be a condition that fracture occurs when a specified mechanical condition is satisfied in the local damage region around a crack. As the criterion, the energy and the local stress conditions [1-3] in this region or the hybrid theory which is taken into account for both conditions [4] have been proposed. There are corresponding local damage regions in brittle fracture, fatigue fracture and creep fracture, respectively. Each scale of these local damage regions is different respectively. For example, each local damage region is considered to correspond with the plastic region [5] for fatigue fracture and creep damage region [6] for creep fracture. For brittle fracture, the end region or process region are considered to be the dominating region of fracture [7]. However, its physical meaning has not yet been clarified. Under this condition, determination of this local region using the experimental fracture toughness has been conducted and the scale of this region is considered to be that of several grains [8]. The process region is considered to be a disordered region of atomic arrangement [3] which concerns fracture of atomic bond. This analysis requires the effects of crack, dislocations and atoms of which scales are order of $\mathrm{mm}, \mu \mathrm{m}$ and $\mathrm{nm}$ on local stress field around a crack or dislocation groups. Since it is necessary to construct two dimensional atomic arrangements to realize a modeled crack or dislocations, conduction of this analysis by atomic mechanics alone requires large scale and high accuracy numerical analyses.

Under this background, authors aim to show the possibility of conducting the analysis of determination of local dominating region of brittle fracture by convenient scale of numerical analysis such as PC level analysis to conduct various calculations conveniently.

The concept of this analysis is as follows.

(1) The macro and micro local stress fields such as a crack and a dislocation are introduced as mechanical boundary conditions of the region of analysis. It causes the elimination of the necessity of the establishment of two dimensional atomic models, which gives the validity of conducting one dimensional analysis.

(2) By utilizing property of potential system of atomic force, instead of using actual atoms array, super atoms array is considered since the mechanical similarity is valid between them. Super atom 
has mechanical equivalence with several actual atoms. Under the boundary condition of (1), an array of these super atoms was considered and a convenient mechanical analysis which obtains mechanical equilibrium positions of super atoms was conducted.

(3) Furthermore, these super atoms were sequentially dispersed under the mechanically equivalent state. Consequentially, the number of these atoms was increased and at each stage of the corresponding scale of these atoms, the mechanical equilibrium positions of these atoms were numerically analyzed.

(4) The characteristic of self-similarity of atom arrays was investigated based on the fractal analysis through each stage of the scale of super atoms. Super atoms were sequentially dispersed and the mechanical equilibrium positions of these atoms were numerically analyzed.

(5) The numerical analyses were conducted up to the scale stage of the super atomic array which shows the characteristic of self-similarity. From this scale stage of super atoms, disordered region of atomic arrays around the crack tip or dislocation with the actual scale of atomic array was predictively calculated and this region was defined as the process region of fracture.

Using this theory, the characterization of disordered region in the macroscopic local stress field around the crack tip becomes possible conveniently and with high accuracy. It enables us to theoretical prediction of the process region which is the dominant region of brittle fracture without the aid of experimental results.

\section{The Model and Method of Analysis}

\subsection{The Model of Analysis}

The concept of super atoms and dislocations which represent mechanically equivalent several actual atoms and dislocations, that is, the concept of image atoms and dislocations were used. Since stress field around a dislocation and interactive force between atoms are conservative, mechanical similarity on scale will be valid. Therefore, similar equations of stress field and interactive forces of dislocation and atoms of which values of intensity are different are adopted for super atoms and dislocations. At each scale stage of super atoms, using equations of interactive forces which have corresponding intensity, the equilibrium positions of super atoms were numerically analyzed. The flow of analysis and the concept of mechanical equivalence between super and actual atoms were shown in Fig. 1. With increment increase in the number of atoms, interactive forces between these atoms were dispersed and the equilibrium positions of atoms in the array were numerically analyzed respectively. The fractal analyses were conducted for the morphology of distribution of atomic density in the array at each scale stage of super atoms and the existence of self-similarity of the atoms array was investigated through each scale stage. When the characteristic of the self-similarity appears through each scale, the projection method is applied to the sequential changing characteristics of the morphology of distribution of atomic density to predict the equilibrium distribution of actual atomic arrays by considering similarity of the changing characteristic being kept due to the existence of the self-similarity. The dominating local region of brittle fracture was predicted by this method and results obtained was compared with previous results [8]. 


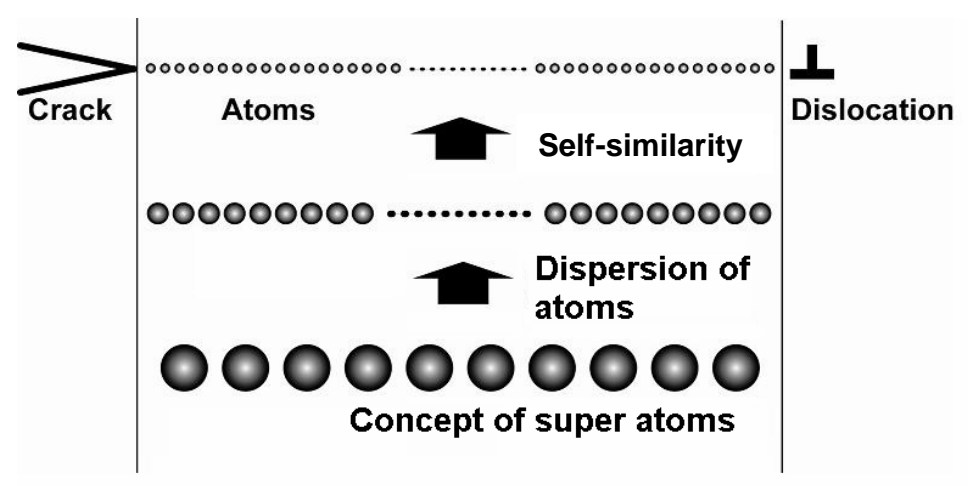

Figure 1. Dispersion method from super atoms to actual atoms under the mechanical equivalence (Scaling from macro to micro under the conservative mechanical system)

\subsection{Basic Equation}

In this analysis, the number of $N$ of super atoms were placed between a crack and a super dislocation which represents dislocation groups. The equations of interaction forces exerted on super atoms were mechanically considered to be symmetry with those exerted on actual scale atoms [9]. The mechanical model used for this analysis was shown in Fig. 2. A crack tip exists at the site of $x=0$ and shearing stress was exerted parallel to the crack that is mode II condition. The super dislocation exists at the site of $x=d$. The number of $n$ of super atoms were placed the same scale interval. In this analysis, to avoid the jump out of atoms from this region during the process of analysis of obtaining the equilibrium positions of atoms between the crack and the super dislocation, fixed atoms were placed at the site of the crack tip and the super dislocation respectively. Local stress fields by the crack and super dislocation were given by boundary conditions in this analysis. The interaction forces between these local stress fields and fixed atoms were neglected.

The interaction force exerted on each super atoms due to the crack, super dislocation and other super atoms in the array was given by Eq. (1),

$$
I_{i}^{*}=\sum_{\substack{j=0 \\ j \neq i}}^{N+1} \frac{4 \varepsilon}{\sigma}\left\{12\left(\frac{\sigma}{x_{i}-x_{j}}\right)^{13}-6\left(\frac{\sigma}{x_{i}-x_{j}}\right)^{7}\right\}+\frac{K_{I I}}{\sqrt{2 \pi x_{i}}}-\frac{n A^{*}}{\left(x_{d}-x_{i}\right)}
$$

Where $i=1 \sim(N-1), \varepsilon$ : constant with dimension of energy, $\sigma:$ constant with dimension of length, $K_{I I}:$ stress intensity factor of mode II which concerns local stress of the crack tip, $x_{i}$ : the position of the $i$ th atom, $n:$ intensity of the super dislocation, $A^{*}:$ intensity of an isolated actual dislocation. The first and second terms of right hand side of Eq. (1) were interactive forces due to other super atoms exerted on the $i$ th atoms. The third and the forth terms of right hand side of Eq. (1) were interactive forces due to local stress fields of the crack and super dislocation exerted on the $i$ th super atom.

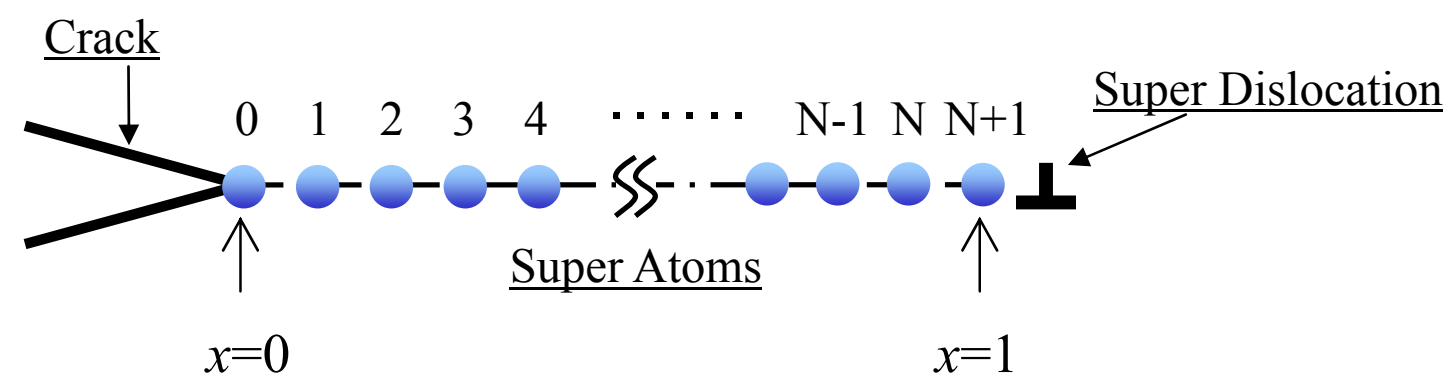

Figure 2. The mechanical equilibrium model of a crack, a super dislocation and super atoms 


\subsection{The algorithm of mechanical dispersion of atom groups}

The value of $x^{+}{ }_{i, n}$ is taken as the non-dimensional variable given by Eq. (2). $\sigma$ is defined as $l$. Concerning the dispersion up to the scale of atom, $1 /(\beta \mathrm{N})^{13}$ and $1 /(\beta \mathrm{N})^{7}$ was exerted to the first and the second terms of Eq. (1) respectively to decrease and disperse interactive forces between super atoms with increase in the number of atoms. By converting Eq. (1) using Eq. (2), the non-dimensional representation of Eq. (1) was given by Eq. (3). That is, $1 /(\beta \mathrm{N})$ is an adjusting parameter of the reasonable intensity of potential field corresponding with each scale of atoms.

$$
x_{i, n}^{+}=\frac{x_{i, n}}{l} \quad, \quad \sigma=l
$$

Where, $x^{+}$is non-dimensional position of atom, $\beta$ is a dispersion coefficient of super atoms. $l$ is a representative length and it equals to value of $\sigma$.

$$
I_{i, n}^{*}=4 \varepsilon \sum_{\substack{j=1 \\ j \neq i}}^{N}\left\{\frac{12}{(\beta N)^{13}} \frac{1}{\left(x_{i, n-1}^{+}-x_{j, n-1}^{+}\right)^{13}}-\frac{6}{(\beta N)^{7}} \frac{1}{\left(x_{i, n-1}^{+}-x_{j, n-1}^{+}\right)^{7}}\right\}+\frac{K_{I I} \sqrt{l}}{\sqrt{2 \pi x_{i, n-1}^{+}}}-\frac{n A^{*}}{\left(x_{d}^{+}-x_{i, n-1}^{+}\right)}
$$

\subsection{Method of analysis}

On the basis of the basic equation, to obtain equilibrium positions of super atoms, following two methods were adopted.

\subsubsection{Direct method}

Equation (2) represents forces exerted on each super atom. When value of $I_{i}{ }^{*}$ is not zero, each super atom moves toward the corresponding equilibrium position dominated by Eq. (4).

$$
M \frac{d^{2} x_{i, n}}{d t^{2}}=I_{i, n}^{*}
$$

Where $n$ is the step number when corresponding atom moves step by step toward the equilibrium position under the Eq. (3).

By Eq. (4), velocity of each atom at the time incremental value, $\Delta t$ is given by Eq. (5). Therefore, moving distance during the time incremental value, $\Delta t$ is given by Eq. (6). Using Eq. (6), the position of each atom at the time of $(t+\Delta t)$ is given by Eq. (7). Where $M$ is an imaginary mass and for convenience from the view point of calculation, $M$ was taken as unity.

$$
\begin{aligned}
V_{i, n} & =I_{i, n}^{*} \Delta t+V_{i, n-1} \\
\Delta x_{i, n} & =V_{i, n} \Delta t \\
x_{i, n+1} & =x_{i, n}+\Delta x_{i, n}
\end{aligned}
$$

This method [9] can be applied to any cases of moving distance of atoms, however the calculation time of numerical analysis becomes longer as compared with Verlet method.

\subsubsection{Verlet method}

On the basis of Taylor expansion, Verlet method was proposed to obtain numerical solutions for the type of Eq. (4) with high accuracy and short calculation time. This method is mainly applied to molecular dynamics. This method is summarized as follows.

On the basis of Taylor expansion, finite difference representation of two order derivative was given by Eq. (8). 


$$
x(t+\Delta t)+x(t-\Delta t)=2 x(t)+(\Delta t)^{2} \frac{d^{2} x}{d t^{2}}
$$

Using Eq. (8), $x_{i, n+1}$ is given by Eq. (9).

$$
x_{i, n+1}=2 x_{i, n}-x_{i, n-1}+(\Delta t)^{2} I_{i, n}^{*}
$$

In this paper, these two methods were adopted to conduct this numerical analysis and results obtained by these methods were compared from the view point of accuracy, the number of atoms which can be calculated and calculation time of numerical analysis.

The converged conditions on the equilibrium positions of atoms were given by Eqs. (10) and (11).

$$
\begin{gathered}
\left|\frac{x_{i, n+1}-x_{i, n}}{x_{i, n}}\right|<10^{-9} \\
\sum_{i=1}^{N}\left|I_{i, n}^{*}\right| / N<10^{-4}
\end{gathered}
$$

The center position of atomic distance and atomic density and the changing rate of atomic density to the initial atomic density were given by Eqs. (12)-(14).

$$
\begin{aligned}
& X_{i}=\frac{x_{i-1}+x_{i}}{2} \\
& d_{i}=\frac{1}{x_{i}-x_{i-1}} \\
& D_{i}=\frac{d_{i}-d_{i, 0}}{d_{i, 0}}
\end{aligned}
$$

The center position of atomic density was shown in Fig. 3. The positive value of $D$ means that the distance between neighboring atoms expands due to local stress field. The region with negative value of $D$ was defined as the disordered region of atomic array and it is related to the process region which dominates brittle fracture $[7,8]$. Conditions of analyses were shown in Table 1.

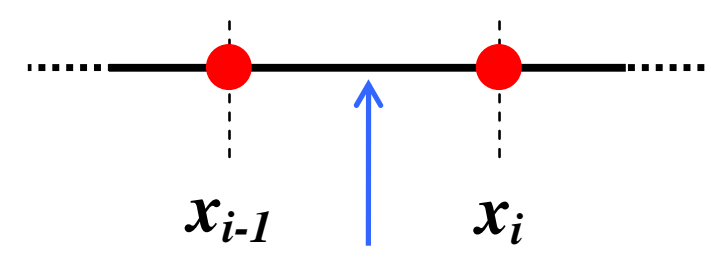

The center position of atomic distance

Figure 3. The definition of the center position of atomic distance

Table 1. Conditions of analyses

\begin{tabular}{|c|c|c|c|}
\hline & Condition1 & Condition2 & Condition3 \\
\hline $\begin{array}{c}K_{I I} \\
\left(\mathrm{MPam}^{1 / 2}\right)\end{array}$ & 20 & 40 & 20 \\
\hline$n A^{*}(\mathrm{MPa})$ & 0.049 & 0.049 & 0.1 \\
\hline$N$ & \multicolumn{3}{|c|}{$\begin{array}{c}10 \sim 1000 \text { (Direct method) } \\
10 \sim 3000 \text { (Verlet method) }\end{array}$} \\
\hline
\end{tabular}


Analyses were conducted for the following cases.

(1) The case of local stress by the crack and the super dislocation being equivalent at the center position of $(0,1)$. (Condition 1 )

(2) The case of local stress by the crack being two times larger than that of super dislocation at the center position of $(0,1)$. (Condition 2$)$

(3) The case of local stress by the crack being half of that of super dislocation at the center position of $(0,1)$. (Condition 3$)$

Since the stress singularity of the crack against the distance from the crack tip $\left(r^{-1 / 2}\right)$ is smaller than that of super dislocation $\left(r^{-1}\right)$, the high stress region of the former is wider than that of the latter.

\subsection{Fractal analysis based on Box counting method}

In this paper, fractal analysis was adopted to estimate the self-similarity of the distributed characteristics of $D$ based on Box counting method.

\section{Results of Analysis}

\subsection{The comparison of two methods and the characteristics of changing rate of atomic density, D}

The effects of atomic scale and number on the characteristics of $D$ defined by Eq. (14) were shown in Figs. 4(a), (b) and (c) under three conditions.

Results obtained by both of the direct and Verlet methods showed the same characteristics of atomic distributions that atomic density increase at the center position of analysis region, $(0,1)$ and decrease at positions of both ends, that is the crack tip and the super dislocation. The direct method can be applicable for any case of the moving distance of atom, however the latter is based on the Taylor expansion and the small moving distance is required. Furthermore, the calculating time of the former is much longer than that of the latter as shown in Fig. 5.

In the region of $D$ having a negative value as shown in Figs. 4 (a), (b) and (c), the breaking stress of atomic bond was applied. Therefore, this region is considered to be a dominating region of fracture. As shown in Figs. 4(a), (b) and (c), with increase in the effect of the crack or the super dislocation, correspondingly, the occurrence of the region of $D$ having negative value near the crack or dislocation becomes typical.

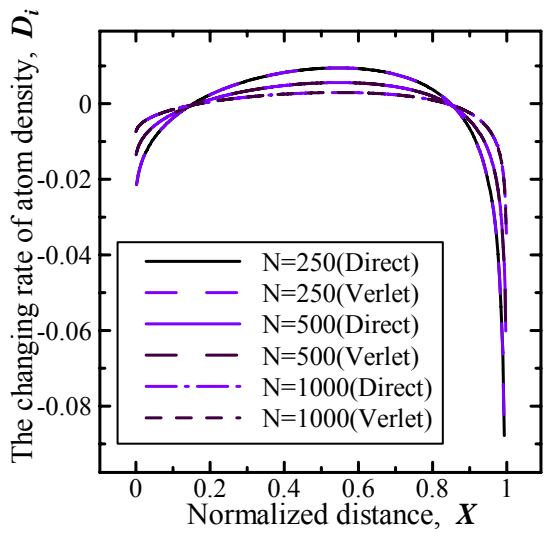

(a) Condition 1

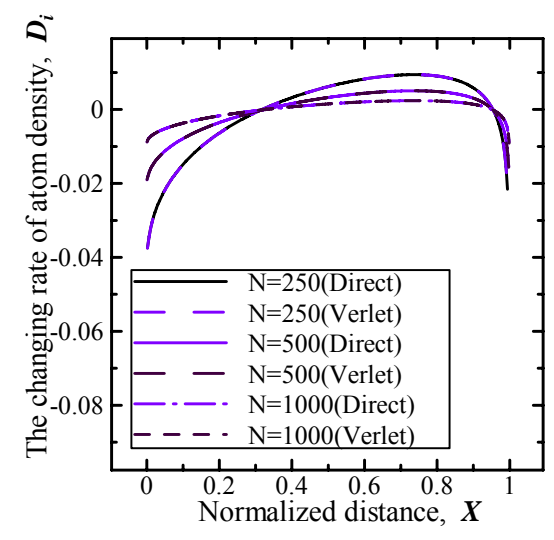

(b) Condition 2

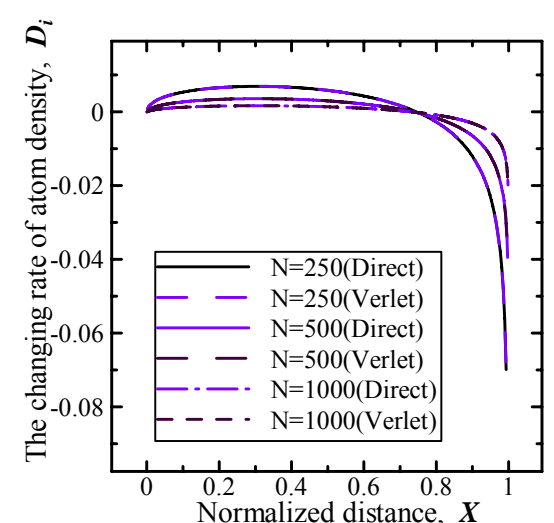

(c) Condition 3

Figure 4. Plots of changing rate of atomic density $D$ vs. normalized distance on each condition 


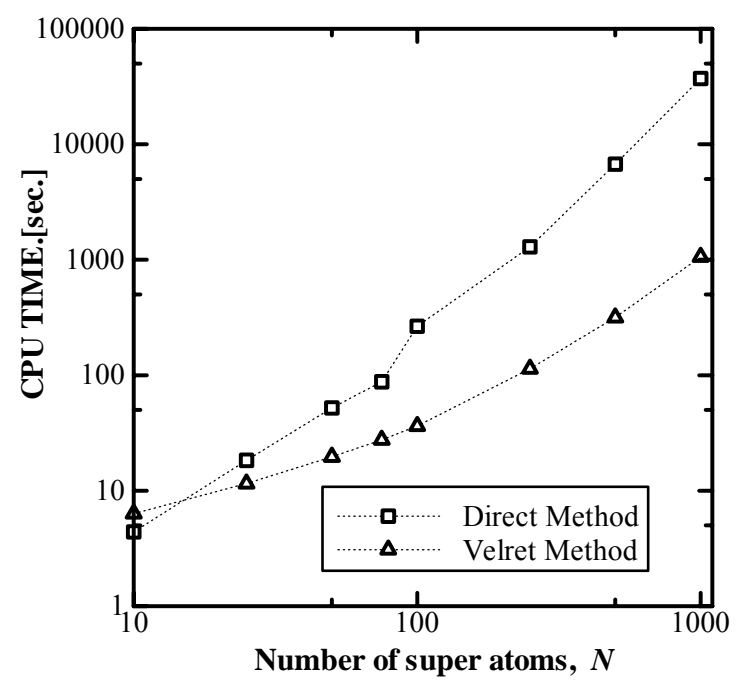

Figure 5. Plots of calculating time vs. number of super atoms on each method

\subsection{Fractal analysis of the distribution of $D$ values}

On the basis of Box counting method, fractal dimensional values, $F D$ were calculated and they were plotted against the number of super atoms as shown in Figs. 6 (a), (b) and (c). These results show that $F D$ takes a specified saturated constant value when the number of super atoms is larger than $N_{c}$ $=250$.

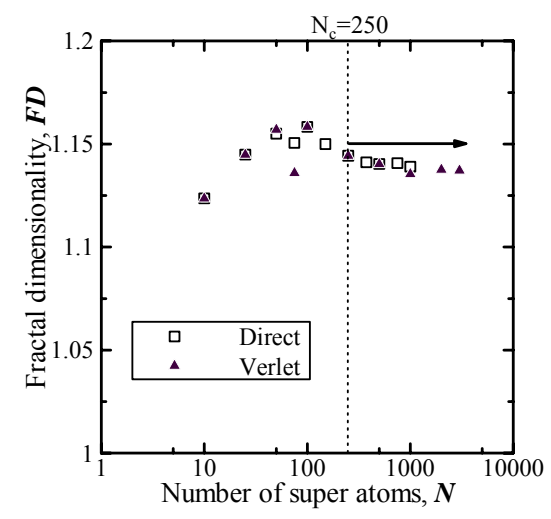

(a) Condition 1

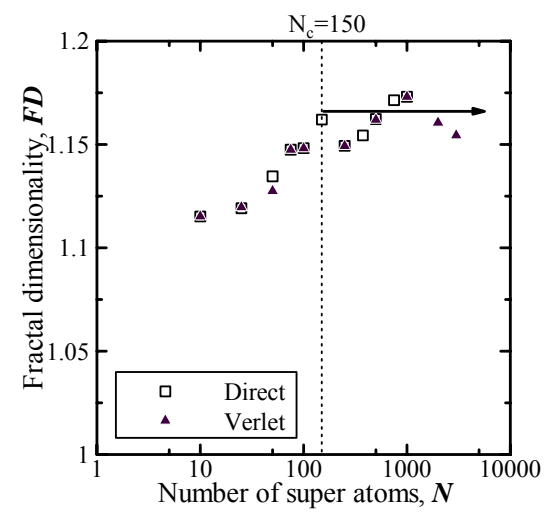

(b) Condition 2

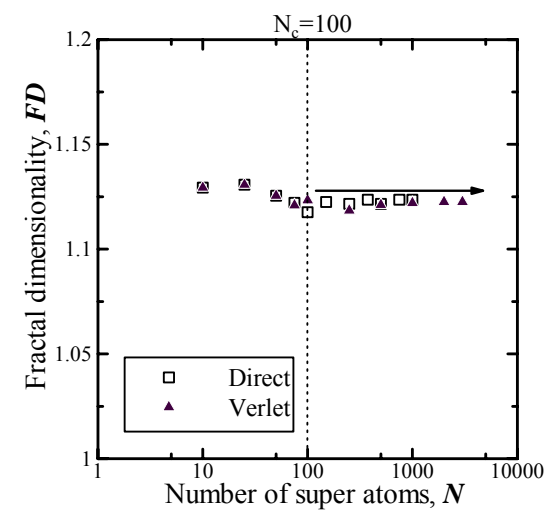

(c) Condition 3

Figure 6. Plots of fractal dimensional value vs. the number of super atoms on each condition

\subsection{Determination of dominating region of fracture using projection method}

To predict the dominating region of fracture, the region where a value of $D$ takes negative value is defined as the disordered regions of super atoms. As shown in Fig. 7, they were characterized by $L$ at the side of the crack and $G$ at that of super dislocation. The changing characteristics of $L$ and $G$ were plotted against the number of super atoms as shown in Figs. 8 (a), (b) and (c). Both of characteristics of $L$ and $G$ were found to take constant values, respectively in the region where the distribution of $D$ shows fractal characteristics. 


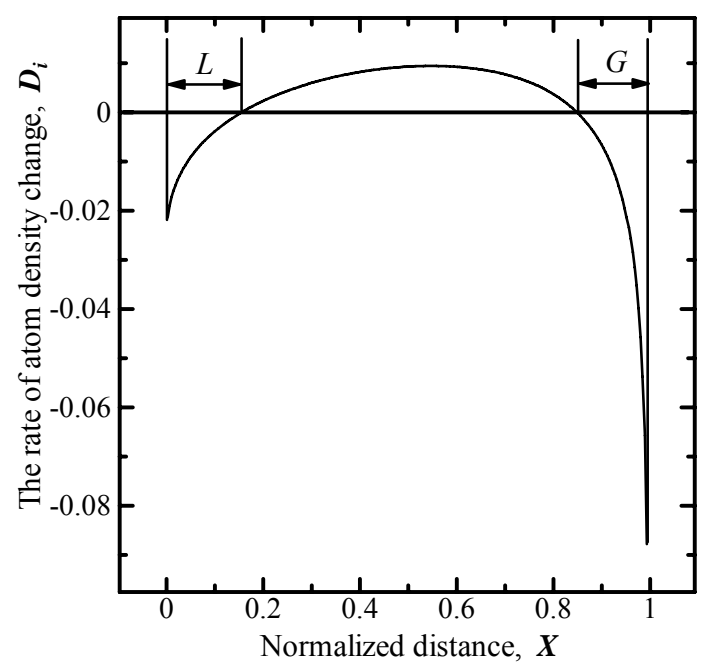

Figure 7. Definition of disordered regions of super atoms

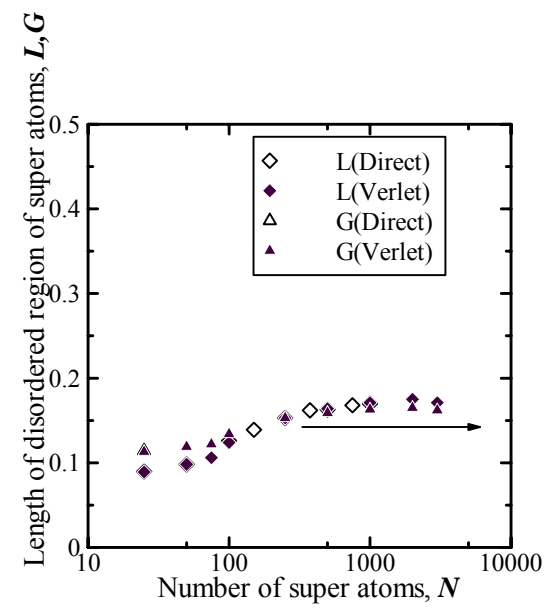

(a) Condition 1

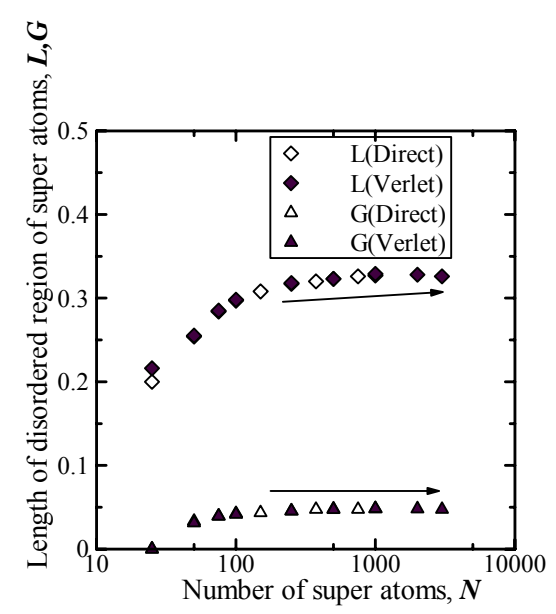

(b) Condition 2

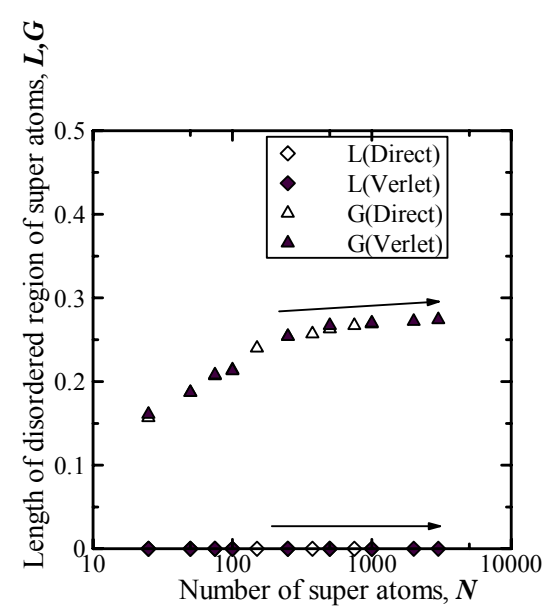

(c) Condition 3

Figure 8. Plots of the length of disordered regions of super atoms vs. the number of super atoms on each condition

\section{Considerations}

These fractal analyses show that self-similarity of the distribution of super atoms is considered to be held when $N$ is larger than $N_{c}\left(N_{c}=250\right)$. Using this property and projection approach, it becomes possible to predict the actual arrangement of atoms. The region of analysis is assumed to be the average value of experimental values of trigger point for $\mathrm{Ni}-\mathrm{Cr}-\mathrm{Mo}-\mathrm{V}$ steel, that is, $10 \sim 210 \mu \mathrm{m}$ [10]. When actual distance between neighboring atoms is assumed to be $0.3 \mathrm{~nm}$, the number of atom, $N$ is considered to be $3 \times 10^{5}$. Based on Fig. 8, values of $L$ and $G$ at $N=3 \times 10^{5}$ were predicted as shown in Table 2 .

Table 2. Disordered region at $N=3.0 \times 10^{5}$

\begin{tabular}{|c|c|c|c|c|c|c|}
\hline & \multicolumn{3}{|c|}{ Direct method } & \multicolumn{3}{c|}{ Verlet method } \\
\cline { 2 - 7 } & Condition 1 & Condition 2 & Condition 3 & Condition 1 & Condition 2 & Condition 3 \\
\hline$L$ & 0.238 & 0.384 & 0.0 & 0.211 & 0.359 & 0.0 \\
\hline$G$ & 0.203 & 0.062 & 0.398 & 0.183 & 0.055 & 0.420 \\
\hline
\end{tabular}


These results show that the length of $L$ and $G$ are 20,30 and $40 \mu \mathrm{m}$ under three conditions, respectively. These values were almost equal to that of grain size for Ni-Cr-Mo-V steel and it is in good agreement with the dominating region of fracture obtained experimentally by Ritchie [8].

Therefore, our proposed method is found to well predict theoretically the dominating factor of fracture.

\section{Conclusions}

(1) On the basis of the analytical method using the concepts of super atoms, super dislocation and self-similarity, the atomic distribution from super atoms to those with small scale was found to show the self-similarity and the possibility of analyzing the behaviors of actual atoms using projection method was validated.

(2) The direct method and Verlet method were found to give the same results on the atomic distribution, however calculating time is different.

(3) Using our proposed method, the dominating region of fracture was shown to derive and it was in good agreement with that obtained experimentally by Ritchie.

\section{References}

[1] G.R. Irwin, Handbuch der Physik, Springer 6 (1958) 551.

[2] J.R. Rice, Int J Frac Mech, 2 (1966) 426.

[3] T. Yokobori, An Interdisciplinary Approach to Fracture and Strength of Solids, 1968, Wolters-Noordhoff Pub. The Netherlands, T. Yokobori, Zairyo kyoudogaku, The $2^{\text {nd }}$ Edition (1974) Iwanami shoten, In Japanese.

[4] T. Yokobori, S. Sawaki, S. Nakanishi, Engng Fract Mech, 12 (1979) 125-141.

[5] T. Yokobori, A.T. Yokobori, Jr., J of the Japan Institute of Metals, 42 (1978) 88-95.

[6] A.T. Yokobori, Jr., T. Kuriyama, Y. Kaji, Advances in Fracture Research, Proc of ICF10, in the content of Special Lecture of CD-rom, Honolulu 2001, Elsevier Science (2001).

[7] K.B. Broberg, Cracks and Fracture, Academic Press, (1999).

[8] R.O. Ritchie, J.F. Knott, J.R. Rice, J Mech Phys Solids, 21 (1973) 395.

[9] A.T. Yokobori, Jr., T. Ohmi, Y. Nagumo, D. Yoshino, Proc. of the $50^{\text {th }}$ Japan National Symposium Strength, Fracture and Fatigue (2006) 57-60.

[10]J. Watanabe, T. Iwadare, Y. Tanaka, T. Yokobori, Engineering Fracture Mechanics, 28, (1987) 589. 